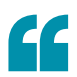

this study uncovered novel mutations and potential new therapeutic targets.

\title{
Multiple myeloma in the spotlight
}

Identifying the acquired somatic mutations in a tumour is a powerful approach for elucidating the molecular mechanisms of cancer progression. A study recently published in Nature describes the full genome sequencing analysis of multiple myeloma, a cancer of mature B lymphoid cells. In addition to confirming the involvement of genes that had previously been associated with multiple myeloma development, this study uncovered novel mutations and potential new therapeutic targets.

Multiple myeloma is thought to result from the activation of $\mathrm{MYC}$, FGFR3, KRAS, NRAS and the nuclear factor- $\kappa \mathrm{B}$ pathway $(\underline{\mathrm{NF}-\kappa \mathrm{B}}$ pathway) and is characterized by chromosomal abnormalities, including translocations and trisomies. To better define the mechanism of transformation a team led by Todd Golub completed whole-genome sequencing (WGS) of 23 patient samples and whole-exome sequencing (WES) of 16 patient samples and compared each tumour to its corresponding normal counterpart. They identified 2.9 tumour-specific point mutations per million bases, including an average of 35 amino acid-altering point mutations and 21 chromosomal rearrangements per sample.

The authors next examined the incidence of protein-altering mutations and identified ten genes that showed significant mutation rates, including several that were already implicated in multiple myeloma. Six genes were identified that were not previously known to be involved in cancer. Two of these genes, DIS 3 and FAM46C, are thought to be involved in protein homeostasis, and the authors further revealed that 16 of 38 ( $42 \%)$ of the patients exhibited mutations in genes the products of which facilitate RNA processing, protein translation or the unfolded protein response, processes that have not previously been linked to multiple myeloma.

Interferon regulatory factor 4 (IRF4), a transcription factor that is involved in plasma cell differentiation, and its target PRDM1 also contained biologically significant mutations, as did BRAF. Gene set analyses identified point mutations and structural rearrangements in 11 NF- $\kappa B$ pathway genes, thereby supporting previous evidence for NF- $\kappa \mathrm{B}$ signalling in multiple myeloma progression. Interestingly, genes involved in histone modification and blood clot formation were also implicated.

By studying 38 cancer genomes, the authors have revealed the complexity of pathway deregulation that facilitates multiple myeloma progression. The investigation provides an unprecedented level of detail that would not have been possible through single genome analysis, and has catalysed the discovery of many novel mutations and therapeutic targets that warrant further investigation and may be applicable to other cancers.

Mhairi Skinner, Consulting Editor, NCI-Nature Pathway Interaction Database

ORIGINAL RESEARCH PAPER Chapman, M. A et al. Initial genome sequencing and analysis of multiple myeloma. Nature 471, 467-472 (2011)

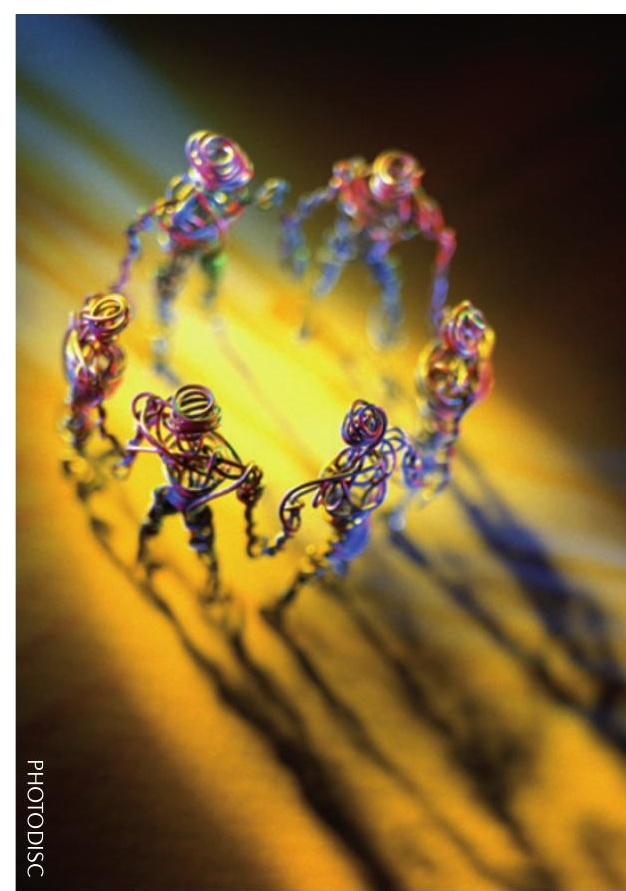

